
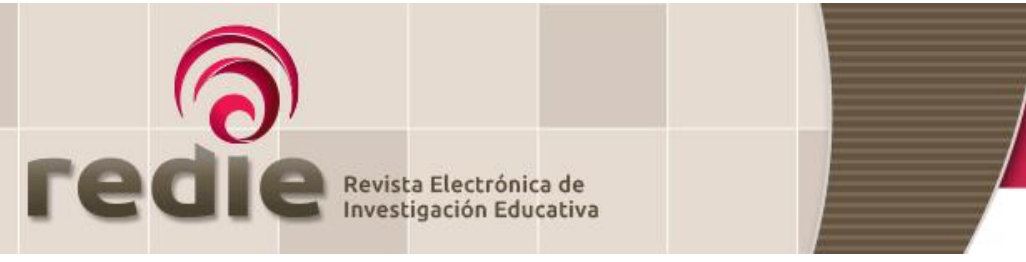

\title{
Emociones y sentido de autoeficacia de los futuros profesores de inglés
}

\section{Emotions and Sense of Self-Efficacy of Pre-Service English Teachers}

Maria del Rosario Reyes-Cruz (*) https://orcid.org/0000-0001-8780-1502

(*) Universidad de Quintana Roo

(Recibido: 28 de octubre de 2018; Aceptado para su publicación: 4 de marzo de 2019)

Cómo citar: Reyes-Cruz, M. R. (2020). Emociones y sentido de autoeficacia de los futuros profesores de inglés. Revista Electrónica de Investigación Educativa, 22, e25, 1-14. https://doi.ora/10.24320/redie.2020.23.e25.2686

\section{Resumen}

El objetivo de este trabajo fue analizar la variación de los estados emocionales y del sentido de autoeficacia de los profesores de inglés en formación a largo de sus prácticas docentes, establecer la relación entre ambos constructos y determinar la fuente de activación de los estados emocionales. Se utilizó un diseño cualitativo exploratorio en el que participaron diez futuros profesores de inglés. Los hallazgos indican que todos los practicantes experimentaron estados emocionales negativos al inicio de las prácticas y que la activación provenía del hecho de impartir clases. El sentido de autoeficacia inicial era bajo y aumentó paulatinamente a lo largo de las prácticas. Las emociones también evolucionaron de negativas a positivas en ese lapso. Las emociones negativas parecen generar un sentido de autoeficacia bajo del mismo modo que las positivas generan un sentido de autoeficacia alto.

Palabras clave: Afectividad, eficacia del docente, enseñanza de idiomas, práctica pedagógica.

\begin{abstract}
The aim of this study was to explore changes in the emotional states and sense of self-efficacy of trainee English teachers in their teaching internship, establish the relationship between these two constructs, and determine the trigger of these emotional states. A qualitative exploratory design was employed to conduct the study, which involved ten pre-service English teachers. The findings indicate that all student teachers experienced negative emotional states at the beginning of their internship, which were triggered by the fact they were giving classes. Their sense of self-efficacy was initially low, but gradually increased over the course of their intership. Their emotions also shifted from negative to positive over the same period. Negative emotions appear to produce a low sense of self-efficacy in the way positive ones lead to a high sense of self-efficacy.
\end{abstract}

Keywords: Emotions, language teaching, self-efficacy, teaching practice.

\section{Introducción}

Por mucho tiempo, los estados emocionales no fueron considerados como un tema relevante para la educación porque en palabras de Robertson (2018) "nos han enseñado, infructuosamente, a reprimir nuestros afectos y emociones en deferencia hacia nuestro intelecto" (p. 13). Recientemente se ha empezado a estudiar la influencia de los estados emocionales sobre los procesos cognitivos y 
motivacionales de los profesores. No obstante, existe aún poca literatura que investigue la influencia de estos en las creencias de autoeficacia para la enseñanza (Hernández, 2017), a pesar de que las investigaciones han demostrado que el sentido de autoeficacia influye en el tipo de prácticas pedagógicas que los profesores realizan (Choi y Lee, 2018). De igual manera, se sabe que los profesores con un mayor dominio emocional propician un mejor clima en el aula (Lavy y Eshet, 2018) y son más estratégicos para afrontar problemas en el contexto escolar (Yin et al., 2017).

En el caso de los profesores en formación, la literatura indica que los cursos y actividades de práctica docente son un espacio muy importante, dado que en ese contexto los practicantes tienen contacto por primera vez con situaciones docentes reales (Fajardo y Miranda, 2015) y empiezan a construir su identidad como profesores (Fletcher y Kosnik, 2016). No obstante, las prácticas docentes también pueden convertirse en espacios que evidencien inconsistencias entre la formación teórica y la realidad escolar (Morales, 2016). En México se imparten 125 programas de licenciatura relacionados con la enseñanzaaprendizaje del inglés (Asociación Nacional de Universidades e Instituciones de Enseñanza Superior, 2016). Es decir, existen cientos de estudiantes en el país preparándose para ser profesores de este idioma.

A pesar de lo antes dicho, no fue posible encontrar estudios nacionales que investigaran algún aspecto de las prácticas de enseñanza que normalmente deben realizar los estudiantes en formación en este tipo de programas. El último análisis sobre la investigación en lenguas extranjeras en México (Ramírez, 2013) indica que el $7 \%$ de la investigación nacional tiene como objeto de estudio la formación docente. No obstante, al revisar con detalle los reportes por estado se verificó que prácticamente todos investigan diversas problemáticas de profesores en servicio y los profesores en formación pasan prácticamente inadvertidos.

En México se enseña inglés en la Secundaria desde hace varias décadas y en Primaria de manera oficial a partir de 2009, a través del Programa Nacional de inglés para la Educación Básica (Ramírez et al., 2014). El argumento principal de la actual Estrategia Nacional de inglés es que se trata de "una política de equidad, inclusión y justicia, ya que el inglés tiene que ser parte del derecho a una educación de calidad" (Notimex, 2017). No obstante, existen críticas sobre la calidad de la enseñanza del inglés en México. Las razones argüidas son variadas; una de las principales es la baja preparación de los profesores (O’Donogue, 2015). Es importante entonces incrementar la investigación de los procesos de formación de los futuros profesores de inglés (En adelante FPI).

El presente estudio tuvo como objetivo analizar la variación de los estados emocionales y del sentido de autoeficacia de los FPI a largo de sus prácticas docentes, establecer la relación entre ambos constructos y determinar la fuente de activación de los estados emocionales.

El sentido de autoeficacia se define como "la creencia del profesor en su capacidad para organizar y ejecutar las acciones necesarias para realizar con éxito una tarea de enseñanza específica en un contexto determinado" (Tschannen-Moran et al., 1998, p. 233). En la literatura especializada se utilizan los términos: creencias de autoeficacia (Martins et al., 2015), sentido de autoeficacia (Chacón y Chacón, 2010) y sentimiento de autoeficacia (Covarrubias y Mendoza, 2015) como sinónimos. Los estados emocionales, por su parte, se conciben como "formas de actuación personalmente representadas y socialmente construidas, que emergen de juicios conscientes o inconscientes relacionados con la percepción de éxito рara alcanzar metas, mantener estándares o creencias durante las interacciones personales en un determinado contextos socio-histórico" (Schutz et al, 2006, p. 344).

Respecto a los FPI y su sentido de autoeficacia sólo se encontraron dos estudios: Cocca y Cocca (2016) hallaron disparidad en el sentido de autoeficacia de los profesores según sus experiencias de desempeño, y Reyes y Murrieta (2017) obtuvieron el mismo resultado. Los datos del segundo estudio revelaron que la mayoría de los participantes se siente muy eficaz en la enseñanza. No obstante, se encontraron inconsistencias entre la autoeficacia percibida, el nivel de inglés y las experiencias previas de desempeño, este hecho sugiere una calibración inadecuada de las creencias de autoeficacia. 
En el contexto internacional, las investigaciones sobre los FPI son también exiguas. Algunos hallazgos han mostrado que los futuros profesores albergan creencias positivas sobre su autoeficacia (Külekçi, 2011; Tagle et al., 2012). Los factores que influyen sobre estas creencias son: la cultura y el contexto (Berg y Smith, 2014), el diálogo con pares y la experiencia (Liaw, 2009). En contraparte, se ha encontrado que la falta de entrenamiento sobre conocimientos pedagógicos y de contenido del inglés repercute en un bajo sentido de autoeficacia, aunque la experiencia obtenida durante las prácticas lo incrementa (Filatov y Phil, 2015).

Estudios como los de Kim y Cho (2014) indican que las creencias en una autoeficacia alta y la motivación intrínseca disminuyen el impacto del shock que experimentan los practicantes al enfrentarse a un contexto de enseñanza real, y que la investigación-acción es una herramienta valiosa para desarrollar el sentido de autoeficacia, el aprendizaje autónomo y la capacidad para resolver problemas (Cabaroglu, 2014). Las investigaciones concuerdan en que a mejor desempeño como profesor mayor sentido de autoeficacia en la enseñanza, y viceversa.

En cuanto a los estados emocionales de los FPI, en México sólo se localizó el estudio de Mugford et al. (2015), quienes encontraron que los practicantes experimentan un amplio abanico de emociones tanto negativas como positivas, aunque la mayoría enfatiza estas últimas y trata de sublimar las primeras. En el ámbito internacional no se detectaron trabajos cuyos participantes fueran FPI. Tampoco se localizaron trabajos que investigaran al mismo tiempo el sentido de autoeficacia docente de los FPI y su relación con los estados emocionales.

Los elementos empíricos derivados de esta investigación pueden contribuir a mejorar los currículos de los programas de formación dado que estos otorgan poca o nula atención a las emociones y al sentido de autoeficacia (Andrade, 2016). La literatura revisada muestra la importancia de desarrollar en los futuros profesores un manejo emocional (Mugford et al., 2015) y un sentido de autoeficacia (Martins et al., 2015) adecuados, que confluyan en una docencia crítico-reflexiva.

\subsection{La autoeficacia percibida y los estados emocionales}

Esta investigación se basa en la teoría de la autoeficacia de Bandura (1997), quien define el sentido de autoeficacia como "la creencia en la propia capacidad de organizar y ejecutar las acciones necesarias para alcanzar los logros deseados" (p. 3), y afirma que los desempeños complejos, como es el caso de la enseñanza, son construcciones organizadas y controladas en su mayoría por habilidades cognitivas y autorregulatorias. El conocimiento de las reglas y estrategias de la docencia para determinar cursos de acción permite a los profesores manejar las exigencias de su quehacer diario.

Según Bandura (1997) existen cuatro fuentes de información que generan el sentido de autoeficacia en las personas: la experiencia previa, la experiencia vicaria, la persuasión verbal y los estados fisiológicos y emocionales. El presente estudio se enfoca en la última fuente, en particular en los estados emocionales. Estos indicadores somáticos son especialmente importantes en áreas que involucran lidiar con estresores. Las personas pueden interpretar la activación emocional en situaciones estresantes como signos de debilidad o de disfunción. La información que estos estados emocionales proporcionan no es en sí misma un diagnóstico de la eficacia real, pero la afecta mediante el procesamiento cognitivo que se hace de ella.

El contexto ejerce una influencia importante en la forma en que se interpretan los estados emocionales internos. Luego entonces, el efecto en dichos estados será diferente dependiendo de los factores situacionales y el significado que las personas les otorguen. Diferentes interpretaciones de la agitación interna tendrán diferentes impactos en la autoeficacia percibida (Bandura, 1997). Los participantes de este estudio se desarrollan en el contexto de sus prácticas docentes. Las emociones que experimenten en esa circunstancia y la interpretación que les atribuyan los harán elaborar un juicio sobre qué tan capaces son.

La información somática que los FPI reciben es interpretada como preceptos y éstos funcionan como base para juzgar su eficacia personal. Este juicio normalmente se hace a partir de la activación autónoma 
percibida y no sobre la eficacia real. Por lo general una agitación moderada aumenta la atención y facilita el despliegue de las habilidades, pero una agitación elevada entorpece la calidad del funcionamiento (Bandura, 1997). Los individuos, y por ende los FPI, varían en cuanto al origen de su agitación emocional, en la forma de interpretarla y en la manera en que ésta afecta su sentido de autoeficacia.

\section{Método}

El estudio se efectuó en una institución pública de educación superior ubicada en el sureste de México. Esta universidad imparte una licenciatura cuyo objetivo es formar profesores de inglés. En el plan de estudios existen dos cursos de práctica docente (noveno y décimo semestres) cuya finalidad es preparar a los estudiantes para el ejercicio de la docencia. Los lugares en los que se realizan las prácticas son escuelas primarias o secundarias con las que la institución tiene convenios. Dependiendo del número de practicantes y los lugares disponibles, en ocasiones los practicantes pueden elegir el nivel educativo en el que deseen realizarlas.

El diseño adoptado para la investigación fue cualitativo exploratorio debido a que, hasta donde se tiene conocimiento, los estados emocionales y su relación con la autoeficacia de los FPI no han sido estudiados conjuntamente en el ámbito internacional ni en el nacional. McNeill (2018) considera que es importante realizar investigaciones de este tipo para entender el porqué de fenómenos que no han sido claramente explicados y poder sentar bases firmes para el futuro desarrollo de constructos sólidos e intervenciones creativas.

La selección de los participantes se realizó mediante criterio (Creswell y Creswell, 2018). Los estudiantes debían haber terminado ya su primera práctica y estar cursando la segunda. Se invitó a participar voluntariamente a todos los que cumplían con el criterio. La muestra final estuvo constituida por cinco hombres y cinco mujeres cuyas edades se encontraban en un rango de 21 a 23 años. El instrumento principal fue la guía de entrevista semiestructurada (ver Apéndice) puesto que facilita la compresión del significado que los participantes adscriben a sus experiencias (Seidman, 2013).

Los participantes fueron informados de los objetivos del estudio, del uso exclusivamente académico de la información proporcionada y de que se les asignaría un pseudónimo para proteger su identidad (Creswell y Creswell, 2018); otorgaron su consentimiento informado y designaron fecha para realizar las entrevistas según su disponibilidad. Se efectuó una entrevista en profundidad de entre 60 y 90 minutos a todos los participantes; en algunos casos fue necesaria una segunda entrevista para aclarar dudas, la cual tuvo una duración aproximada de entre 30 y 50 minutos.

El análisis se realizó siguiendo las estrategias propuestas por Bernard et al. (2017): descubrir códigos y subcódigos, describir sus elementos centrales y periféricos, organizarlos jerárquicamente, reducir los códigos a familias y relacionarlos con el marco teórico. El primer ciclo de codificación estuvo guiado por tres códigos prestablecidos por la teoría: estados emocionales, fuente de activación, y sentido de autoeficacia. En el segundo ciclo de codificación (Saldaña, 2016) se refinaron los códigos; como resultado, los códigos teóricos se convirtieron en familias dado que agrupaban a un número mayor y más específico de códigos. Por ejemplo, los estados emocionales congregaban el terror, la felicidad y el nerviosismo.

La validación del análisis se realizó mediante la revisión de las trascripciones e interpretaciones por parte de los participantes (Creswell y Creswell, 2018). También se analizaron los dos programas de estudio de las materias relacionadas con las prácticas para contrastar lo dicho por los participantes con los documentos oficiales. Se puso particular atención a los temas abordados, las actividades realizadas, su duración y los productos a entregar. 


\section{Resultados y discusión \\ 3.1 La variación de los estados emocionales y su fuente de activación}

En cuanto a la variación de los estados emocionales, se halló que al inicio de las prácticas el estado más frecuentemente experimentado por los FPI fue el nerviosismo (7 participantes). Por ejemplo: "Más que nada eran nervios, a veces me bloqueaba mucho, llegábamos al salón y tenía todo preparado, sabías todo lo que ibas a hacer y llegabas y los nervios hacían que se te olvidara todo" (Participante 2). Una estudiante experimentó terror: "La primera práctica fue más angustia y terror. No la quería hacer [la práctica] porque tenía miedo, sentía que no estaba lista" (Participante 1). Mientras que otros participantes tuvieron emociones menos fuertes, como inhibición: "Mi tono de voz era muy bajo por lo mismo, porque me inhibía, me daba pena" (Participante 7), o inseguridad: "Inseguridad sobre todo porque eran chicos ya grandes [sic] ellos sí te podían juzgar y decir: creo que el maestro no sabe" (Participante 9).

Las experiencias arriba descritas por los participantes muestran que la enseñanza es un proceso emocional en sí mismo (Zembylas, 2005). Los estudios indican que estas primeras experiencias influyen en su forma de concebir la profesión (Suárez y Basto, 2017) y en la identidad que construyan como profesores (Anttila et al., 2016).

Bandura (1997) establece que la intensidad de las emociones varía según los individuos debido a diversos factores, pero que siempre se relaciona con qué tan capaces se sienten en ese momento. Normalmente una agitación elevada, como el terror, produce una sensación de incapacidad que suele desembocar en un bajo desempeño.

La fuente que con mayor recurrencia activaba emociones negativas era precisamente impartir la clase. La Participante 3 señaló que dar las primeras clases "la ponía muy nerviosa". Los aspectos específicos de esta actividad eran diversos; los participantes mencionaron: a) la presencia de las maestras titulares de grupo: "Luego te están viendo los maestros y se pueden empezar a reír" (Participante 2); b) la propia reacción emocional: "Lo que más me preocupaba era cómo iba yo a reaccionar si un alumno no me hacía caso" (Participante 3); c) la falta de control de grupo: "Esos niños tienen serios problemas de disciplina y sí fue todo un reto estar calmándolos" (Participante 5), y d) la poca preparación inicial: "Fue más que nada porque no me sentía bien preparado [para dar la clase]" (Participante 7).

Los practicantes se encontraban en un contexto desconocido, desarrollando una actividad compleja en la que no se sentían suficientemente capaces; además de que eran observados por los estudiantes y los profesores titulares del grupo. Lo anterior provocó en ellos distintos niveles de agitación (Bandura, 1997), todos ligados a emociones negativas. Los resultados coinciden con los de Arizmendi et al. (2016), obtenidos también en contexto mexicano. Los estudiantes se encontraban en la etapa denominada "preocupación por sí mismos" (Fuller y Brown, 1975), en donde el centro de atención eran ellos y sus circunstancias y no tanto la calidad de su enseñanza.

Hacia el final de la primera práctica y al inicio de la segunda, algunos estudiantes experimentaron también emociones negativas, pero en este caso la fuente de activación era externa. Los estudiantes señalaron: a) a las profesoras titulares: "Las otras maestras [titulares del grupo] atrasaban a los niños al irles a lavar las manos, me molestaba que no respetaban nuestra hora, nuestro tiempo" (Participante 1); b) las actitudes de los estudiantes: "Me sentía triste también porque, digo, estoy aquí hablando y nadie me hace caso" (Participante 6), y c) la falta de aprendizaje: "Me desespera que no aprendan y sí me siento un poco angustiada cuando veo que en los exámenes no entienden" (Participante 2).

La experiencia de desempeño (Bandura, 1997) ganada durante la práctica permitía a los estudiantes ejercer un cierto grado de dominio de sus emociones y enfocar su atención en los estudiantes. Ma y Cavanagh (2018) encontraron también que los practicantes creen tener baja eficacia en el manejo de grupo, mientras que los hallazgos de Ryan (1986) indican que los profesores se culpan por la falta de aprendizaje de sus estudiantes. A estas alturas de la práctica, algunos estudiantes se encontraban en la etapa de "preocupaciones acerca de las situaciones o tareas" mientras que otros avanzaban hacia la de 
"preocupaciones por el impacto en los estudiantes" (Fuller y Brown, 1975).

Durante la segunda práctica, las emociones fueron tornándose positivas. La mayoría de los estudiantes dijo sentirse con mayor confianza: "Ya después se me pasó, ya me siento más confiado" (Participante 7); varios experimentaron satisfacción: "Es muy satisfactorio ver cómo te responden los estudiantes" (Participante 2); mientras pocos practicantes manifestaron emociones intensas como la felicidad: "Me sentía muy feliz, me gustaba dar la clase a los niños" (Participante 3).

Las fuentes que activaban estas emociones positivas fueron: a) la mejora en la planeación de la clase: "Ahora tu lesson plan te sale la mayoría de las veces como tú lo planeaste" (Participante 2); b) resolver dudas: "Me sentía contenta de ayudar a mis estudiantes a resolver sus dudas" (Participante, 1); c) constatar el aprendizaje de los estudiantes: "Ellos aprenden lo que tú estás haciendo y te sientes satisfecho" (Participante 5), y d) las muestras de afecto de los estudiantes: "Ya el último día que fui, pues, cantamos, bailamos y todo eso en inglés y ya luego una niña hizo un pequeño dibujo de una rosa y ya me lo dio, me sentí feliz" (Participante 5).

La experiencia obtenida durante los meses de práctica permitió a los participantes sentirse más eficaces. Percibir el avance de sus estudiantes retroalimentó su sentido de autoeficacia. Recibir el afecto de los niños igualmente validó su forma de enseñar. Bandura (1997) reconoce a la experiencia y la persuasión (verbal y no verbal) como dos factores que influyen en las emociones experimentadas. Los resultados concuerdan con los de Hascher y Hagenauer (2016) y Dávila et al. (2015), quienes hallaron que las emociones se tornaban positivas después de los seminarios de prácticas. Al final de éstas, la mayoría de los participantes parecían encontrarse en la etapa de "preocuparse por el impacto en sus estudiantes".

\subsection{El sentido de autoeficacia y su variación a lo largo de las prácticas}

Respecto a la variación del sentido de autoeficacia de los participantes durante las prácticas, la mayoría dijo no sentirse muy capaz en la primera, básicamente debido a la falta de preparación pedagógica para enseñar a niños; una participante expresó:

Sí teníamos la idea de que vamos a enseñar niños; mas no teníamos una capacitación en sí para hacerlo y eso es lo que estaban diciendo: ¿Por qué no antes de mandar a los estudiantes a Práctica Docente I, por qué no se da alguna materia sobre Pedagogía infantil? Sería de muy buena ayuda para nosotros. En el momento que estás ahí te das cuenta de que todo ese proceso de enseñar a niños es especial. (Participante 4).

Al preguntar a los participantes si observaban diferencias en su eficacia en la segunda práctica respecto de la primera, 9 de los 10 señalaron haber mejorado. Las razones fueron diversas. Algunos hicieron sus prácticas en un nivel distinto al de la primera y les fue mejor en este último nivel. La Participante 6 aseveró: "Los niños me cuestan trabajo, en cambio en la secundaria, con los adolescentes me va mejor. Me considero muy buena en secundaria. Siento que tengo mejor control de grupo y creo que los alumnos aprenden".

Otros practicantes repitieron la experiencia de dar clases a niños. El Participante 2 mencionó: "Ahora ya conozco más cómo son los niños y ya tengo mejor control de grupo, y mayor seguridad; eso hace que mis clases sean más efectivas" (Participante 2).

Bandura (1997) establece que las creencias de autoeficacia son de contexto específico y que la posibilidad de trasladarlas de un contexto a otro depende de la especificidad o generalidad que las personas les otorguen. Impartir clases en primaria o en secundaria es fundamentalmente diferente dadas las etapas de desarrollo en las que los estudiantes se encuentran. Los practicantes que se adaptaron mejor a uno de los niveles repitieron, y los que no, utilizaron la estrategia de probar un contexto distinto.

Sólo el Participante 7 dijo no sentirse mucho más eficaz respecto de la primera práctica: "Creo que no he 
mejorado mucho desde que empezamos con las prácticas, siento que hay cosas que me fallan, si lo llego a hacer [trabajar como profesor] más que nada sería por necesidad" (Participante 7). Esta situación puede deberse a la falta de conocimientos pedagógicos, lo mismo encontraron Filatov y Phil (2015).

En cuanto al nivel de eficacia percibido por los participantes días antes de terminar el segundo curso de práctica, 7 dijeron sentirse muy capaces. La Participante 1, por ejemplo, apuntó: "Creo que soy muy buena maestra" (Participante 1). Estos resultados coinciden con los de Külekçi (2011), Reyes y Murrieta (2017) y Tagle et al. (2012), quienes también descubrieron que los profesores en formación albergan creencias positivas sobre su eficacia docente. No obstante, se ha encontrado que los profesores en servicio, y en este caso parece aplicar también para los FPI, tienden a subestimar la complejidad de la tarea (Bandura, 1997), dado que se encuentran en lo que Ryan (1986) denomina etapa de "fantasía". En esta etapa, los practicantes aún no han estado completamente solos a cargo de un grupo ni por un tiempo prolongado. Esta aparente sobrevaloración podría obstaculizar la capacidad de autorreflexión y las aspiraciones de mejora (Wheatley, 2002).

Tres estudiantes se autoevaluaron como moderadamente eficaces. El Participante 7, por ejemplo, comentó: "Siento que no tengo una alta capacidad para enseñar, me falta todavía más" (Participante 7). Estos tres estudiantes parecen tender a reflexionar más sobre su práctica. Los estudios han encontrado que esta disposición ayuda a mejorar el manejo del salón de clase (Voss et al., 2017) y que un sentido de autoeficacia moderado puede conducir a la mejora de la enseñanza mediante la autorreflexión (Babaei y Abednia, 2016).

\subsection{La influencia de los estados emocionales en la percepción de autoeficacia}

La influencia de los estados emocionales fue muy fuerte al inicio de las prácticas. Siete de los estudiantes dijeron sentirse nerviosos al impartir sus primeras clases. Los tres restantes comentaron haber sentido terror, inseguridad y nerviosismo. El Participante 2 comentó: "Las emociones negativas influyen mucho cuando vas a dar tu clase te sientes tan... te ganan los nervios. Al menos yo sentí que no di lo mejor como maestro y siento que en algunas clases no aprendieron los alumnos" (Participante 2).

En la mayoría de los casos las emociones fueron cambiando, conforme avanzaban las prácticas los estados emocionales positivos empezaron a prevalecer y el sentido de autoeficacia a aumentar. La participante 3 explicó:

Bueno, al sentirme ya en las otras semanas [quiere decir al contar con más semanas de práctica], me sentí relajada, sentí que eso me daba más ganas de hacerlo mejor. Igual los niños te hacen sentir bien, feliz. Todas esas emociones influyeron para que diera bien la clase y que aprendieran realmente. (Participante 3).

Estos resultados coinciden con los de Moafian y Ghanizadeh (2009) y Rastegar y Memarpour (2009), quienes también hallaron relación entre las creencias de autoeficacia de los profesores y su manejo de las emociones. Adicionalmente, también hubo practicantes que dijeron que los estados emocionales son variables y que es algo inherente a ser profesor: "Entonces, es normal que un profesor de inglés tenga momentos buenos y malos, emociones positivas y negativas según las clases y las etapas de su vida" (Participante 3).

Vale la pena destacar que aquellos participantes que al final de las dos prácticas se evaluaron como altamente capaces fueron también los que dijeron haberse sobrepuesto más rápidamente a sus emociones negativas y cuyo nivel de activación era moderado. Bandura (1997) asevera que los estados emocionales son variables y que la interpretación y control de los mismos influye de manera importante en el sentido de autoeficacia. Efectivamente, la enseñanza involucra emociones tanto negativas como positivas (Isenbarger y Zembylas, 2006, p. 124), en especial para los profesores en formación, controlarlas puede resultar particularmente difícil. 


\subsection{Los programas de estudio}

Como forma de validación, se contrastó lo manifestado por los participantes con los programas de estudio de las prácticas docentes, en la tabla I se observan los objetivos, así como las actividades y su duración.

Tabla I. Aspectos relevantes de los programas de prácticas

\begin{tabular}{|c|c|c|c|c|}
\hline \multicolumn{5}{|c|}{ Primera práctica docente } \\
\hline Objetivos & Contenidos & Actividades & Duración/cantidad & Producto \\
\hline \multirow{4}{*}{$\begin{array}{l}\text { - Aplicar } \\
\text { conocimientos } \\
\text { previos } \\
\text { - Analizar textos } \\
\text { - Observar clases } \\
\text { - Organizar y } \\
\text { diseñar planes de } \\
\text { clase y materiales } \\
\text { - Impartir clases. }\end{array}$} & \multirow{4}{*}{$\begin{array}{l}\text { - Las observaciones } \\
\text { - Principios del enfoque } \\
\text { comunicativo } \\
\text { - Estilos de aprendizaje } \\
\text { - Las inteligencias múltiples } \\
\text { - Diseño de clases para niños } \\
\text { - Manejo de grupo } \\
\text { - La disciplina } \\
\text { - La estructura de una lección } \\
\text { de lengua }\end{array}$} & Observaciones & $\begin{array}{l}5 \text { (1 por nivel } \\
\text { educativo) }\end{array}$ & Reporte \\
\hline & & $\begin{array}{l}\text { Diseño de } \\
\text { planes de clase }\end{array}$ & $\begin{array}{l}12 \text { de } 1 \text { hora } \\
\text { o } 2 \text { de } 6 \text { horas }\end{array}$ & Planes de clase \\
\hline & & $\begin{array}{l}\text { Práctica con } \\
\text { grupos reales }\end{array}$ & 12 horas & $\begin{array}{l}\text { Autorreflexión } \\
\text { escrita por clase } \\
\text { impartida }\end{array}$ \\
\hline & & Clínicas & 12 horas & $\begin{array}{l}\text { Reflexión } \\
\text { colectiva }\end{array}$ \\
\hline \multicolumn{5}{|c|}{ Segunda práctica docente } \\
\hline Objetivos & Contenidos & Actividades & Duración/cantidad & Producto \\
\hline \multirow{3}{*}{$\begin{array}{l}\text { - Aplicar } \\
\text { conocimientos } \\
\text { previos } \\
\text { - Observar clases } \\
\text { - Organizar y } \\
\text { diseñar planes de } \\
\text { clase y materiales } \\
\text { Impartir clases }\end{array}$} & \multirow{3}{*}{$\begin{array}{l}\text { Enseñanza en cinco niveles } \\
\text { educativos (jardín de niños, } \\
\text { primaria, secundaria, } \\
\text { preparatoria, universidad) }\end{array}$} & Observaciones & 8 horas & Reporte \\
\hline & & Microenseñanza & $50-75$ minutos & $\begin{array}{l}\text { Cometarios } \\
\text { verbales }\end{array}$ \\
\hline & & $\begin{array}{l}\text { Práctica con } \\
\text { grupos reales }\end{array}$ & 20 horas & $\begin{array}{l}\text { Reflexión } \\
\text { colectiva }\end{array}$ \\
\hline
\end{tabular}

La tabla I muestra la ausencia de temas como el papel de las emociones en la enseñanza, la autoeficacia docente y el modelado de la docencia. Si bien es cierto que se contempla la observación de clases, no se explicita la exposición sistemática y teóricamente sustentada a modelos de diversos tipos. Bandura (1997), por ejemplo, argumenta que los modelos de alto desempeño tienen efectos diferenciados de los modelos de afrontamiento, según sean observados por personas novatas o experimentadas.

Aunque temas como la disciplina, el manejo de grupo y la enseñanza específica en diversos niveles educativos se incluyen en los programas, e incluso la enseñanza a niños aparece en ambas materias, no está claro cuánto tiempo se dedica a los mismos ni el nivel de profundidad. A juzgar por los comentarios de los participantes, estos temas parecen tratarse de manera muy general y en un tiempo limitado. De igual forma, los participantes no mencionaron nada respecto a las reflexiones colectivas y las autorreflexiones contempladas en los programas, dando pie a pensar que éstas no tuvieron una influencia importante en sus prácticas.

Adicionalmente, ambos programas parten de la presunción de que los estudiantes cuentan con conocimientos teóricos y procesuales sobre un vasto número de temas sobre la enseñanza de lenguas que han sido estudiados años atrás. Esta suposición es errada, pues no se puede saber a ciencia cierta qué tanto han retenido los estudiantes de los contenidos pasados y, por ende, sobre qué bases se están fundando las prácticas docentes. De igual manera, el número de horas de práctica es muy bajo (32 en total). Esto sugiere que los estudiantes no tienen suficiente tiempo para contar con experiencias más ricas y variadas. 


\section{Conclusiones}

El objetivo de este trabajo fue analizar la variación de los estados emocionales y del sentido de autoeficacia de los futuros profesores de inglés a largo de sus prácticas docentes, establecer la relación entre ambos constructos y determinar la fuente de activación de los estados emocionales. Los hallazgos indican que el sentido de autoeficacia creció paulatinamente a lo largo de las prácticas, de la misma forma que los estados emocionales evolucionaron de negativos a positivos durante las mismas. Las emociones negativas iniciales se correspondían también con un bajo sentido de autoeficacia mientras que, de igual manera, las emociones positivas experimentadas con mayor frecuencia hacia el final de las prácticas se relacionaban con un sentido de autoeficacia mayor al inicial.

Al inicio la activación de las emociones negativas era generada por factores internos, como la escasa preparación inicial; posteriormente, la activación provenía de factores externos, como el poco respeto de las profesoras de grupo hacia la clase de inglés. Respecto de las emociones positivas, éstas eran activadas por el aprendizaje de los estudiantes y la mejora en el propio desempeño.

También es de notar que aquellos practicantes que se sobrepusieron más rápidamente a sus estados emocionales negativos fueron los mismos que, después de dominar las emociones cuya fuente era la preocupación por la propia actuación, siguieron experimentando emociones negativas -pero esta vez debido a factores externos que dificultaban el aprendizaje de los estudiantes. Estos mismos participantes fueron quienes dijeron experimentar las emociones positivas más fuertes al percibir el afecto y logros de sus estudiantes.

La literatura previa concuerda con los hallazgos de este estudio respecto a que la experiencia incrementa el sentido de autoeficacia (Cocca y Cocca, 2016; Filatov y Phil, 2015; Liaw, 2009) y que los practicantes normalmente albergan creencias positivas sobre su eficacia docente (Külekçi, 2011; Tagle et al., 2012). Los resultados sobre las emociones sólo son comparables con el trabajo de Mugford et al. (2015), quienes también encontraron un amplio rango de emociones experimentado por los practicantes. Respecto a la relación de las emociones y el sentido de autoeficacia, los resultados coinciden con los de Moradkhani y Haghi (2017), quienes también hallaron que las emociones negativas se relacionan con un sentido de autoeficacia bajo y viceversa. En lo que concierne las fuentes de activación emocional, los hallazgos son similares a los de Anttila et al. (2016), quienes descubrieron que éstas se relacionan con la suficiencia de habilidades docentes y con el cumplimento de las expectativas de enseñanza.

Esta investigación aporta elementos que sugieren la existencia de áreas por entender en la licenciatura donde se forman los participantes. En los programas revisados no se contemplan los temas de autoeficacia ni emociones. Ambos aspectos son fundamentales para los futuros profesores de inglés. Como los datos lo muestran, la mayoría lidió con sus emociones por ensayo y error. Muchos estados emocionales aversivos podrían evitarse si se abordaran estos temas en los cursos. Es vital formar a los estudiantes sobre las estrategias de regulación emocional, mediante un proceso bien diseñado de conocimientos teóricos, procesuales y prácticos. De igual manera, es fundamental diagnosticar qué conocimientos y habilidades reales sobre la docencia poseen los estudiantes antes de iniciar las prácticas. De esta forma se evitaría intentar edificar la formación docente en un terreno, al parecer, débilmente cimentado.

Por otro lado, también se hace patente la necesidad de formación específica en el tema de la enseñanza a niños. En los años recientes existe una tendencia mundial a enseñar inglés desde el nivel preescolar. No obstante, parece que pocas instituciones han alineado sus programas de formación de profesores a dicha política. Luego entonces, los practicantes enseñan en educación básica con escaso conocimiento teórico de cómo los niños aprenden una lengua extranjera. Lo anterior perjudica no sólo el estado emocional y el sentido de autoeficacia de los practicantes, afecta también las experiencias que los niños tienen al iniciarse en el aprendizaje del inglés.

Por último, aunque este trabajo aporta elementos explicativos nunca antes analizados en el área de enseñanza de lenguas extranjeras en contexto mexicano, es importante reconocer que quedan aún 
preguntas por resolver. Habría que profundizar en las historias de vida de los participantes para develar qué otros elementos influyen en aquellos que demostraron mayor compromiso y comportamiento estratégico. De igual forma, sería importante utilizar instrumentos adicionales de recolección de datos, como diarios reflexivos, videograbaciones y observaciones de clase.

\section{Referencias}

Andrade, N. F. (2016). La gustatividad en la formación docente: un fenómeno afectivo emergente. Revista Electrónica de Investigación Educativa, 18(2), 92-04. http://redie.uabc.mx/redie/article/view/817

Anttila, H., Pyhältö, K., Soini, T. y Pietarinen, J. (2016). How does it feel to become a teacher? Emotions in teacher education. Social Psychology of Education, 48(10), 59-63. https://doi.org/10.1007/s11218-016$\underline{9335-0}$

Arizmendi, S., Gillings, B. S. y López, C. L. de J. (2016). How novice EFL teachers regulate their negative emotions. How, 23(1), 30-48. https://doi.org/10.19183/how.23.1.299

Asociación Nacional de Universidades e Instituciones de Enseñanza Superior. (2016). Anuario Estadístico de la Educación Superior 2016-2017. http://www.anuies.mx/informacion-y-servicios/informacion-estadisticade-educacion-superior/anuario-estadistico-de-educacion-superior

Babaei, M. y Abednia, A. (2016). Reflective teaching and self-efficacy beliefs: Exploring relationships in the context of teaching EFL in Iran. Australian Journal of Teacher Education, 41(9), 1-26.

http://ro.ecu.edu.au/ajte/vol41/iss9/1

Bandura, A. (1997). Self-efficacy: the exercise of control. Worth Publishers.

Berg, D. A. G. y Smith, L. F. (2014). Pre-service teachers' efficacy beliefs and concerns in Malaysia, England and New Zealand. Issues in Educational Research, 24(1), 21-40. http://www.iier.org.au/iier24/berg.pdf

Bernard, H. R., Wutich, A. Y. y Ryan, J. W. (2017). Analyzing qualitative data: systematic approaches. Sage.

Cabaroglu, N. (2014). Professional development through action research: Impact on self-efficacy. System, 44, 79-88. https://doi.org/10.1016/j.system.2014.03.003

Chacón, E. J. y Chacón, C. T. (2010). Un modelo para medir el sentido de autoeficacia docente en profesores de inglés como lengua extranjera en secundaria. Revista Evaluar, 10, 1-21.

https://revistas.unc.edu.ar/index.php/revaluar/article/view/456

Choi, E. y Lee, J. (2018). EFL teachers' self-efficacy and teaching practices. ELT Journal, 72(2), 175-186. https://doi.org/10.1093/elt/ccx046

Cocca, M. y Cocca, A. (2016). Self-efficacy in pre-school and primary teachers: does experience, educational background, and teaching level matter? International Conference on Education and New Learning Technologies, Barcelona. https://library.iated.org/view/COCCA2016SEL

Covarrubias, C. G. y Mendoza, M. (2015). Sentimiento de autoeficacia en una muestra de profesores chilenos desde las perspectivas de género y experiencia. Estudios Pedagógicos, 41(1), 63-78.

https://doi.org/10.4067/S0718-07052015000100004

Creswell, J. W. y Creswell, J. D. (2018). Research design: qualitative, quantitative, and mixed methods approaches. Sage. 
Dávila, M, A., Borrachero, A. B., Cañada, F., Martínez, G. y Sánchez, J. (2015). Evolución de las emociones que experimentan los estudiantes del grado de maestro en educación primaria, en didáctica de la materia y la energía. Revista Eureka sobre Enseñanza y Divulgación de las Ciencias, 12(3), 550-564.

https://revistas.uca.es/index.php/eureka/article/view/2942

Fajardo, J. A. y Miranda. I. R. (2015). The paradox of the practicum: affinity to and resistance towards teaching. íkala, Revista de Lenguaje y Cultura, 20(3), 329-341.

https://doi.org/10.17533/udea.ikala.v20n3a04

Filatov, K. y Phil, S. (2015). The relationship between university learning experiences and English teaching self-efficacy: perspectives of five final-year pre-service English teachers. Australian Journal of Teacher Education, 40 (6), 33-59. http://dx.doi.org/10.14221/ajte.2015v40n6.3

Fletcher, T. y Kosnik, C. (2016). Pre-service primary teachers negotiating physical education identities during the practicum. Education, 3(13), 556-565. https://doi.org/10.1080/03004279.2016.1169486

Fuller, F. F. y Brown, O. H. (1975). Becoming a teacher. En K. Ryan (Ed.), Teacher education: $74^{\text {th }}$ yearbook of the National Society of Education (pp. 25-52). University of Chicago Press.

Hascher. T. y Hagenauer, G. (2016). Openness to theory and its importance for pre-service teachers' selfefficacy, emotions, and classroom behavior in the teaching practicum. International Journal of Educational Research, 77, 15-25. https://doi.org/10.1016/j.ijer.2016.02.003

Hernández, V. (2017). Las competencias emocionales del docente y su desempeño profesional. Alternativas en Psicología, 37, 79-92. http://www.alternativas.me/attachments/article/147/06\%20\%20 Las\%20competencias\%20emocionales\%20del\%20docente.pdf

Isenbarger, L. y Zembylas, M. (2006). The emotional labor of caring in teaching. Teaching and Teacher Education, 22(1), 120-134. https://doi.org/10.1016/i.tate.2005.07.002

Kim, H. y Cho, Y. (2014). Pre-service teachers' motivation, teacher efficacy, and expectation of reality shock. Asia-Pacific Journal of Teacher Education, 42(1), 67-81.

https://doi.org/10.1080/1359866X.2013.855999

Külekçi, G. (2011). A study on pre-service English teachers' self-efficacy beliefs depending on some variable. International Online Journal of Educational Sciences, 3(1), 245-260.

http://www.acarindex.com/dosyalar/makale/acarindex-1423904420.pdf

Lavy, S. y Eshet, R. (2018). Spiral effects of teachers' emotions and emotion regulation strategies: evidence from a daily diary study. Teaching and Teacher Education, 73, 151-161.

https://doi.org/10.1016/j.tate.2018.04.001

Liaw, E. (2009). Teacher efficacy of pre-service teachers in Taiwan: the influence of classroom teaching and group discussions. Teaching y Teacher Education, 25(1), 176-180.

https://doi.org/10.1016/j.tate.2008.08.005

MA, K. y Cavanagh, M. S. (2018). Classroom ready? Pre-service teachers' self-efficacy for their first professional experience placement. Australian Journal of Teacher Education, 43(7), 134-151.

http://dx.doi.org/10.14221/ajte.2018v43n7.8

Martins, M., Costa, J. y Onofre, M. (2015). Practicum experiences as sources of pre-service teachers' selfefficacy. European Journal of Teacher Education, 38(2), 263-279.

https://doi.org/10.1080/02619768.2014.968705 
McNeill, C. (junio 2018). Exploratory research: 3 reasons to conduct more of it [mensaje de blog].

https://www.qutcheckit.com/blog/3-reasons-conduct-exploratory-research/

Moafian, F. y Ghanizadeh, A. (2009). The relationship between Iranian EFL teachers' emotional intelligence and their self-efficacy in language institutes. System, 37, 708-718.

https://doi.org/10.1016/j.system.2009.09.014

Moradkhani, S. y Haghi, S. (2017). Context-based sources of EFL teachers' self-efficacy: Iranian public schools versus private institutes. Teaching and Teacher Education, 67, 259-269.

https://doi.org/10.1016/j.tate.2017.06.019

Morales, Y. A. (2016). Unveiling pre-service teachers' attitudes toward teaching: The role of pedagogical practicums. PROFILE Issues in Teachers' Professional Development, 18(2), 47-61.

http://dx.doi.org/10.15446/profile.v18n2.49591

Mugford, G.; Sughrua, W. y López-Gopard, M. (2015).Construction of an English language teacher identity: perceptions and contrasts in Mexico. Mextesol Journal, 39(2), 1-11.

http://mextesol.net/journal/public/files/b10c8a78ad61810720dae2274a1aa0e0.pdf

Notimex. (11 de julio de 2017). Enseñanza del inglés es una política de igualdad: Aurelio Nuño. Excelsior. https://www.excelsior.com.mx/nacional/2017/07/11/1174984

O’Donogue, J. L. (2015). Sorry, el aprendizaje del inglés en México. Mexicanos Primero.

Ramírez, J. L. (Coord.) (2013). Una década de búsqueda: las investigaciones sobre la enseñanza y el aprendizaje de lenguas extranjeras en México (2000-2011). Pearson, Universidad de Sonora, UNAM, Universidad de Colima.

Ramírez, J. L., Sayer, P. y Pamplon, E. N. (2014). English language teaching in public primary schools in Mexico: the practices and challenges of implementing a national language education program. International Journal of Qualitative Studies in Education, 27(8), 1020-1043.

https://doi.org/10.1080/09518398.2014.924638

Rastegar, M. y Memarpour, S. (2009). The relationship between emotional intelligence and self-efficacy among Iranian EFL teachers. System, 37(4), 700-707. https://doi.org/10.1016/i.system.2009.09.013

Reyes, M. R. y Murrieta, G. (2017). Los futuros profesores de inglés: experiencia previa y sentido de autoeficacia. Mextesol Journal, 41(2), 1-17.

http://www.mextesol.net/journal/index.php?page=journal\&id article=2085

Robertson, D. L. (2018). Emotion and professors' developmental perspectives on their teaching. New Directions for Teaching and Learning, 153, 13-23. https://doi.org/10.1002/tl.20277

Ryan, K. (1986). The induction of new teachers. Phi Delta Kappa Educational Foundation.

Saldaña, J. (2016). The coding manual for qualitative researchers. Sage.

Schutz, P. A., Hong, J. Y., Cross, D. I. y Osbon, J. N. (2006). Reflections on investigating emotions in educational activity settings. Educational Psychology Review, 18(4), 343-360.

Seidman, I. (2013). Interviewing as qualitative research. A guide for researchers in Education and the Social Sciences. Teachers College Press. 
Suárez, S. A. y Basto, E. A. (2017). Identifying pre-service teachers' beliefs about teaching EFL and their potential changes. PROFILE Issues in Teachers' Professional Development, 19(2), 167-184.

http://dx.doi.org/10.15446/profile.v19n2.59675

Tagle, T., Del Valle, R., Flores, L. y Ackley, B. (2012). Las creencias de autoeficacia percibida de estudiantes de pregrado de pedagogía en inglés. Revista Iberoamericana de Educación, 58(4), 1-2.

https://rieoei.org/historico/deloslectores/4667Tagle.pdf

Tschannen-Moran, M., Hoy, A. W. \& Hoy, W. K. (1998). Teacher efficacy: its meaning and measure. Review of Educational Research, 68(2), 202-248.

Voss, T., Wagner, W., Klusmann, U., Trautwein U. y Kunter, M. (2017). Changes in beginning teachers' classroom management knowledge and emotional exhaustion during the induction phase. Contemporary Educational Psychology, 51, 170-184. https://doi.org/10.1016/j.cedpsych.2017.08.002

Wheatley, K. F. (2002). The potential benefit of teacher self-efficacy doubts for educational reform. Teaching and Teacher Education, 18(1), 5-22. https://doi.org/10.1016/S0742-051X(01)00047-6

Yin, H., Huang, S. y Lee, J. (2017).Choose your strategy wisely: examining the relationships between emotional labor in teaching and teacher efficacy in Hong Kong primary schools. Teaching and Teacher Education, 66, 127-136. https://doi.org/10.1016/j.tate.2017.04.006

Zembylas, M. (2005). Teaching with emotions: a postmodern enactment. Information Age. 
Apéndice. Guía de entrevista

1. Cuando piensas en las emociones y tus actividades de práctica docente, ¿qué te viene a la mente?

2. ¿Qué emociones experimentaste durante tu primera práctica?

3. ¿Hubo aspectos o situaciones que generaran emociones positivas?

4. ¿Hubo aspectos o situaciones que generaran emociones negativas?

5. ¿Qué tan capaz de impartir clases te considerabas al inicio de las prácticas?

6. ¿Notas alguna diferencia entre tu capacidad para dar clases en la primera práctica y en la segunda?

7. ¿Qué tan capaz de enseñar inglés te sientes actualmente?

8. ¿Hay alguna diferencia en las emociones que experimentas ahora con las que tenías al inicio de las prácticas?

9. ¿Actualmente qué aspectos o situaciones de las prácticas generan emociones en ti?

10. ¿Percibes alguna conexión entre tus emociones y tu capacidad para enseñar? 